\title{
Academic faculty governance and recruitment decisions
}

\author{
Jens Prüfer • Uwe Walz
}

Received: 17 May 2010 / Accepted: 14 September 2011 / Published online: 14 October 2011

(C) The Author(s) 2011. This article is published with open access at Springerlink.com

\begin{abstract}
We analyze the implications of the governance structure in academic faculties for their recruitment decisions when competing for new researchers. The value to individual members through social interaction within the faculty depends on the average status of their fellow members. In recruitment decisions, incumbent members trade off the effect of entry on average faculty status against alternative uses of the recruitment budget if no entry takes place. We show that the best candidates join the best faculties but that they receive lower wages than some lesser ranking candidates. We also study the allocation of surplus created by the entry of a new faculty member and show that faculties with symmetric status distributions maximize their joint surplus under majority voting.
\end{abstract}

JEL Classification D02 $\cdot$ D71 $\cdot$ L22

Keywords Academic faculties $\cdot$ University governance $\cdot$ Status organizations $\cdot$ Labor market competition

\section{Introduction}

In human capital intensive industries recruitment decisions are a crucial organizational task influencing decisively the quality of the main factor of production and, hence, the capabilities and performance of the organization. In many academic faculties recruitment decisions are delegated from the administrators to the academic staff. Masten (2006) shows empirically that this is most pronounced in large research universities, as compared to liberal

\footnotetext{
J. Prüfer $(\bowtie)$

Department of Economics, CentER, TILEC, Tilburg University, P.O. Box 90153, 5000 LE Tilburg, The Netherlands e-mail: j.prufer@uvt.nl

U. Walz

Department of Applied Microeconomics and Management, Goethe University Frankfurt and CFS, Grüneburgplatz 1, 60323 Frankfurt, Germany

e-mail: uwalz@econ.uni-frankfurt.de
} 
arts colleges and more specialized organizations. In research universities the collaboration among faculty members influences research output, which has an effect on the utility of an individual member. Thus, by making recruitment decisions, faculty members decide on their own well-being in the future.

Academic faculties may be viewed in part as "status organizations" in the sense of Hansmann (1986): the value of collaboration among faculty members depends in part on their joint abilities, which can be presumed to be strongly correlated with their external professional status. ${ }^{1}$ Hence, status is considered to be a proxy for the abilities of faculty members (most notably in the research dimension). The higher the status of an individual member, the more valuable this member is for others.

Status is a vertically differentiable good, characterized by some degree of rivalry. The larger the number of members that interact with one person, the less valuable this interaction becomes individually. Although as researchers we experience and participate in recruitment decisions frequently, a theoretical framework that takes into account the specifics of individual collaboration between highly skilled workers is missing.

We concentrate on two questions. First, what are the implications of competition among faculties for the allocation of new researchers? For instance, will top Ph.Ds join the best or just second-tier universities and for what wage? Second, what effects does the entry of a new member have on the joint surplus of incumbent faculty members and the new member himself?

Concentrating on majority voting, we will show that the job candidates (researchers) with the highest status levels join the faculty with the highest average status. Candidates with lower status levels either join the faculty of lesser average status or are not accepted by any faculty at all. New faculty members with low status levels are unable to appropriate any surplus from joining a faculty. In contrast, new members with high status are protected by the competition that arises amongst the faculties and are thereby able to share the surplus with incumbent faculty members. We show that only faculties with symmetric status distributions maximize their joint surplus under majority voting.

We model competition for candidates in a two-faculty framework. The two faculties with a given number of incumbent members with given status levels differ in their average status. A high average status faculty (e.g., Harvard University) competes with a faculty of lower average status (e.g., State University X). Incumbent members trade off the utility they receive from the average status of the other members of their faculty, which has implications for the success of joint research projects, against alternative uses of the hiring budget if no new member enters the faculty. We show that faculty members with low status benefit more from joint research than members with high status. This advantage is diluted more than proportionally by the entry of new members with low status. Hence, we find somewhat surprisingly that low status members are more reluctant to allow candidates to enter the faculty than high status members.

The paper is organized as follows. In the next section we provide an overview of the related literature. In Sect. 3, we outline the baseline model and study competition between two faculties. In Sect. 4, we characterize the equilibrium in this setup and analyze the allocation of surplus. In Sect. 5, we discuss the robustness of our main assumptions; we conclude and sketch alternative applications of our model in Sect. 6. All proofs and alternative model specifications are in the Appendices A and B.

\footnotetext{
${ }^{1}$ In Sect. 6, we outline further applications of our model, such as clubs and international economic organizations (e.g. WTO, ECU).
} 


\section{Related literature}

In order to place our approach in the context of the existing literature we stress upfront the key properties and the main distinguishing features of our model. They are as follows. (i): Status is a one-dimensional, vertically differentiable type variable. Hence, the relative preference ordering of access to a candidate among incumbent members, and vice versa, is equal. All players are endowed exogenously with status, that is, we do not allow them to invest in status. (ii): Making use of the status of a fellow faculty member incurs a cost (for the remaining faculty members) because it dilutes the value of interaction with this fellow, which makes status a rival good. (iii): The actual payoff created by using all faculty members' status differs among individuals because, from the perspective of a lesser ranked member, the average status value of his fellows is greater than from the perspective of a highly ranked member. (iv): The status utility of faculty members is not perfectly transferable as we consider a friction between the payment of money and the consumption of perks.

We can identify several different branches of literature related to our work. First, the seminal works on the economic theory of clubs were published in the 1960s. Most notably, Buchanan (1965) and Olson (1965) initiated a wave of research on the economic theory of clubs and club goods, which was to be further developed in the decades following (see Sandler and Tschirhart 1980, and Cornes and Sandler 1996: Chap. 11). As in Ellickson et al. (1999), we deal with the individual characteristics of new and incumbent faculty members and the interrelation of a faculty's aggregate characteristics and its competition for new candidates. In contrast to those authors, we do not calculate the optimal faculty size explicitly. We do, however, characterize the equilibrium level of wages paid to new candidates. Helsley and Strange (1991) also examine discriminating pricing schemes, but our paper goes further and studies the allocation of surplus within the faculties.

A second strand of the literature covers the dynamic aspects of clubs' decision-making rules and admission of new members. Sobel (2001) focuses on multidimensional characteristics and heterogeneous preferences of members showing the circumstances under which status level thresholds that have to be met by candidates increase or decrease over time. Barbera et al. (2001) and Cai and Feng (2007) offer related approaches analyzing the effects of various interest groups within a club on entrance of new members in equilibrium. Apart from the fact that we do not model dynamic aspects explicitly, our model contrasts with respect to characteristics (i) and partly (iii).

Third, our paper builds on the idea of clubs as status organizations, a notion introduced by Hansmann (1986: 22), who refers to "clubs" as a "prototypical example of status organizations". Hansmann, however, regards the formation of a club system while we assume that clubs - or faculties - already exist and study the competition among them.

The papers most closely related to ours are two by Epple and Romano $(1998,2008)$, who analyze the competition among private and public schools for students in a world where peer effects make school quality dependent on the composition of the abilities of a school's student body. Epple and Romano (1998) argue in their theoretical analysis that competition between tax-financed public schools and profit-maximizing private schools leads the latter to skim off the wealthiest and most able students. ${ }^{2}$ Epple and Romano (2008) study the impact of the design of educational vouchers on the stratification of students across schools and

\footnotetext{
${ }^{2}$ This argument neglects, however, the positive effect that competition between public and private schools has on public-school effectiveness. This competitive effect might potentially overcompensate the sorting effect and lead to higher performance of public schools in a competitive environment. See, for instance, Hoxby (1996) and Couch et al. (1993).
} 
show that, by adding restrictions like tuition constraints, vouchers can capture the benefits without promoting greater stratification of school competition. Masten (1995) is related to these articles as he studies the impact of financial aid coordination among a group of top US universities and argues that the specific characteristics of the higher education industry are difficult to reconcile with traditional cartel theory. We differ significantly by proposing a formal model that analyzes competition among faculties that have similar objective functions and where faculty decisions are determined by incumbent members rather than by a profit-maximizing investor.

Our paper is also closely related to the empirical investigations of governance structures in universities. By looking into the functions and limitations of democratic governance structures in US universities, Masten (2006) shows that democratic decision-making processes are hindered by neither the size of the organization nor the heterogeneity of its voting members. Rather he finds that large universities with heterogeneous departments have more democratic structures in place than small, homogeneous colleges. Against this background he looks into the function of such democratic processes. We complement his analysis to a certain extent. Rather than focusing on democratic decision processes per se, we look into the implications of different (democratic) governance structures. We thereby focus on the input market: the decisions of universities and faculties to hire new faculty members.

\section{The model}

We model two academic faculties, $j \in\{A, B\}$, which compete for a new researcher. Individuals are, with the exception of their status positions, identical. The status position describes their relative value for fellow scholars in social exchange processes and can be attributed to a wide set of characteristics such as methodological and writing skills as well as network relations.

The status positions of incumbent members are drawn from a distribution over the interval $[\underline{s}, \bar{s}]$. Faculty $j$ has $N_{j}$ incumbent members, where $N_{j}$ is a finite number. The member of faculty $j$ with the highest status is called $\bar{n}_{j}$, its member with the lowest status is $\underline{n}_{j}$, and its member with median status is $m_{j}$ (see details below). We assume simple majority voting in both faculties and denote the aggregate status of all incumbent members of faculty $j$ by $\sum_{j} s^{i}$, where $s^{i}$ is the status of member $i$. Setup costs are sufficiently large such that it is prohibitive for a subset of members (or new candidates) to form a third faculty.

Each faculty has access to a given total budget for recruiting new researchers, $T \geq 0$, which it is assigned exogenously by its university's executive board. To focus on the impact of status differences on the competitive outcome, we assume that recruitment budgets in both faculties are the same. Each faculty may offer a new researcher (candidate $C$ ) a wage $W_{j} \leq T$. The faculties are prohibited from distributing the remaining $T-W_{j}$ among existing members. ${ }^{3}$ This restriction implies that the value of a dollar of the residual budget for each member of faculty $j$ is $\alpha T / N_{j}$, where $\alpha \in[0,1)$. In order to facilitate our analysis we assume that $\alpha$ is identical across faculties.

By means of collaboration and social exchange, faculty members can increase their research output and, hence, their well-being. This effect hinges on the average status of the

\footnotetext{
${ }^{3}$ For instance, the remaining budget can be used only to cover departmental operating costs, pay for travel to conferences, the purchase of computer software or datasets, and the like. In many cases, faculties do not increase budgets after failed searches but do get such increases after faculty members have left and the position remains vacant. We summarize these effects in the parameter $\alpha$.
} 
other faculty members, where status is a rival good: each member has a fixed amount of resources (time) to interact with his fellows. If the size of the faculty increases, opportunities to interact with a given colleague decrease, on average. This interpretation of the status variable, as a rival good, is different from status being equal to reputation, a nonrival good. Nonrivalry of status would imply in our setting, that the sum of the status levels of all other members rather than the average status of all other faculty members should be the argument in the individual member's utility function. In the robustness section of our paper, we argue that the paper's main results are unchanged if status is treated as a partially nonrival good. ${ }^{4}$

We assume that, in expectation, collaboration among faculty members is uniformly distributed. Therefore, in expectation, each member gains an equal share of a fellow's time reserved for collaboration..$^{5}$ Cooperation is more productive and valuable for each member the higher is the social status of his/her counterpart. Hence, we depict the utility function of a particular incumbent member $k$ of faculty $j$ as: ${ }^{6}$

$$
u_{j}^{k}=\hat{s}_{j}^{k}+\alpha\left(\frac{T-W_{j}}{N_{j}}\right),
$$

where $\hat{s}_{j}^{k}$ denotes the average status of all the other members in faculty $j$ from the point of view of faculty member $k$. The marginal rate of substitution between status and money is constant for all players and, hence, independent of a member's own status. The introduction of $\alpha$ makes status and money imperfect substitutes. Note that we could have formalized this notion either by assuming concave utility in status or convex operating costs (with respect to the number of faculty members). Introducing $\alpha<1$ instead involves significantly less calculus.

Furthermore, our utility function assumes that every member of faculty $j$ weighs utility from the average status of the faculty by the same factor (technically, we assume an additively separable utility function). Given that there are arguments for deviations in both directions (high status members derive more or less utility from the average status as compared to the low status members), it seems natural to assume identical weights for marginal utility from average status across all faculty members. We discuss relaxing this assumption in Sect. 5.

Before we introduce the new candidate, let us briefly spell out some notation. For member $k$ with status $s^{k}$ in faculty $j$ the average status of all other faculty members is:

$$
\hat{s}_{j}^{k}=\frac{\sum_{j} s^{i}-s^{k}}{N_{j}-1} \text {. }
$$

We assume that faculty $A$ is the more exclusive faculty. That is, the average status in faculty $A$ as well as the status utility enjoyed by the median member is higher than in faculty $B$ :

$$
\hat{s}_{A} \equiv \frac{\sum_{A} s^{i}}{N_{A}}>\frac{\sum_{B} s^{i}}{N_{B}} \equiv \hat{s}_{B} \quad \text { and } \quad \hat{s}_{A}^{m}>\hat{s}_{B}^{m} .
$$

\footnotetext{
${ }^{4}$ See Sect. 5 and Appendix B.1 for details.

${ }^{5}$ In Sect. 5 and Appendix B.3, we analyze the effects of "localized" matching, according to which a member has larger probability of interacting with other members of equal status than with colleagues of higher or lower status. This leads to positive assortative matching. We show that the results of our baseline model are robust to the corresponding change in expected utility.

${ }^{6}$ Subscripts denote faculties, and superscripts denote individuals.
} 
We assume all incumbent faculty members to be immobile because of switching costs. In contrast, the candidates, newly graduated Ph.D.s, for instance, are mobile and can choose to apply at any of the two faculties. ${ }^{7}$ A candidate who is accepted as new member of a faculty affects both arguments of the utility function of the incumbent faculty members. First, by entering faculty $j$ the new candidate, with status value $s^{C}$, changes the average status value enjoyed by incumbent member $k$ to:

$$
\hat{s}_{j}^{k}=\frac{\sum_{j} s^{i}-s^{k}+s^{C}}{N_{j}} .
$$

If candidate $C$ is admitted to a faculty, he also gains via interaction with the other members. Second, he receives a wage $W_{j}$ in faculty $j$. However, by joining a particular faculty, a new member foregoes the benefits, denoted by $R \geq 0$, he would receive from working in another, non-academic profession. As the average status of $j$ 's members from the perspective of a new member equals the average status of the incumbent members, $\hat{s}_{j}$, the utility function of candidate $C$ entering faculty $j$ is:

$$
u_{j}^{C}=\hat{s}_{j}+W_{j}-R .
$$

Note that $W_{j}$ is not discounted by $\alpha$ in the candidate's utility function because there are no rules that prohibit him from spending the wage as he wishes. This is different for incumbent faculty members and reflects institutional rules in most universities.

We model the competition among the two faculties for new entrants as a two-stage game. At the first stage, both faculties $A$ and $B$ simultaneously decide whether they are willing to allow the candidate to enter at all, that is they choose a minimal status level, $s_{j, m i n}$, that the entrant must satisfy. They also make take-it-or-leave-it wage offers, $W_{j}$, to the new candidate. At the second stage, the new entrant can choose to apply to join either of the two faculties that provides him with the greater nonnegative utility and accepts his entry. At both stages of the game, complete information prevails. We solve this game by backward induction for a subgame-perfect solution.

\section{Entry decisions and voting}

Before we turn to the analysis of the details of the decision making process, we need to show that the utility gain from entry of the candidate for incumbent faculty members is strictly monotonic in the member's rank because only then does the median voter theorem apply under majority voting.

The utility differential, that is the post-entry utility minus the pre-entry utility of the $k$-th individual in faculty $j$, is:

$$
\begin{aligned}
\Delta_{j}^{k} & =\frac{\sum_{j} s^{i}-s^{k}+s^{C}}{N_{j}}-\alpha \frac{W_{j}}{N_{j}}-\frac{\sum_{j} s^{i}-s^{k}}{N_{j}-1} \\
& =\frac{s^{C}}{N_{j}}-\frac{\sum_{j} s^{i}-s^{k}}{N_{j}\left(N_{j}-1\right)}-\frac{\alpha W_{j}}{N_{j}},
\end{aligned}
$$

\footnotetext{
${ }^{7}$ We will discuss this assumption further in Sect. 5.
} 
which is strictly increasing in $s^{C}$ and $s^{k}$ (given that the aggregate status in faculty $j$ is held constant). Since a higher $s^{k}$ corresponds to a higher $k$, the utility differential is also increasing in the rank $k$ within the faculty. Therefore we obtain:

Lemma 1 (Admission incentives) Incumbent faculty members with lower status rank gain less from a candidate's entry than members with higher status rank. Thus, the minimal status level of a new member required by an individual incumbent member $k$ is lower the higher is that member's status rank.

Understanding this lemma is key to the remainder of the results of this paper. Without entry the lowest ranking member of faculty $j, \underline{n}_{j}$, enjoys a gross status utility of $\frac{\sum_{j} s^{i}-s(\underline{\underline{n}} j)}{N_{j}-1}$, which is strictly larger than the highest ranking member's, $\bar{n}_{j}$ 's, utility, $\frac{\sum_{j} s^{i}-s\left(\bar{n}_{j}\right)}{N_{j}-1}$. Given that every existing faculty member is willing to vote positively for a prospective new hire as long as it provides a nonnegative utility differential, the highest-ranked member is more liberal than the one with the lowest status. That is, he accepts candidates with less than minimum status (see details in Sect. 4.2). Between the two extremes, gains from collaboration are monotonic. This implies that the median voter theorem applies under majority voting. Hence, we can focus on the pivotal median member for the remainder of the analysis.

Upon the entry of any new member, the advantage of low ranking incumbent members over high ranking members is diluted. Hence, $\underline{n}_{j}$ suffers more than proportionally from entry, which is expressed by (6). The intuition behind this is that the gross benefit from interaction with the new member is equal for all incumbent members. To receive this benefit, however, high status members only give up some interaction with colleagues of comparatively low status. Instead, lower tier members give up some interaction with their high status colleagues. It follows that the relative differential status value of the new hire to incumbent faculty members is increasing in the status rank of the incumbent members. An entrant who participates in social interaction with high status faculty fellows therefore crowds out interactions with incumbent low status members of the faculty.

\subsection{The candidate's decisions}

In the final stage of the game the entrant has to make two decisions: should he join a faculty at all and, if so, which one? The candidate will be willing to join a faculty $j$ if the utility this option offers is positive:

$$
\hat{s}_{j}+W_{j}-R \geq 0
$$

We will refer to this inequality as the participation constraint of the entrant to faculty $j$, $\left(P C_{j}\right)$. It implies that the candidate is willing to enter $j$ if, and only if, the expected gains from interaction with the other faculty members and the wage offered are not lower than his opportunity cost of pursuing a non-academic career.

Given that the newcomer joins either faculty, he will choose the one that offers him the larger net utility, meaning that he will prefer faculty $j$ over faculty $q$ if:

$$
\hat{s}_{j}+W_{j}>\hat{s}_{q}+W_{q} .
$$

If the utilities are equal, which we will refer to as the indifference condition (IC) of the entrant, we will assume that the candidate will join faculty $A$. By using this assumption and 
rearranging (8), we know that faculty $A$ will attract the candidate if the wage it offers and the status advantage it has over faculty $B$ are not smaller than the wage offered by $B$ :

$$
W_{A}=W_{B}-\left(\hat{s}_{A}-\hat{s}_{B}\right) .
$$

Given the anticipated behavior of the entrant, we will now address the optimal behavior of the faculties.

\subsection{The choices of the faculties}

In the first stage of the game, faculties $A$ and $B$ compete by simultaneously choosing a minimal status level for job candidates $\left(s_{j, \min }\right)$ and a wage rate $\left(W_{j}\right)$, taking into consideration the candidate's reaction.

The decision problem of the median member of faculty $j$ is to maximize the utility differential $\Delta_{j}^{m_{j}}$ that he will individually receive from entry of the candidate subject to the candidate's willingness to join faculty $j$ (and not the other faculty, $q$ ):

$$
\begin{aligned}
\underset{s_{j, m i n} ; W_{j}}{\operatorname{Max}} & \operatorname{argmax}\left\{\Delta_{j}^{m_{j}}, 0\right\} \\
& \text { s.t. } \\
& \hat{s}_{j}+W_{j}-R \geq 0, \\
& \hat{s}_{j}+W_{j}>\hat{s}_{q}+W_{q},
\end{aligned}
$$

where

$$
\Delta_{j}^{m_{j}}=\frac{s^{C}}{N_{j}}-\frac{\sum_{j} s^{i}-s^{m_{j}}}{N_{j}\left(N_{j}-1\right)}-\frac{\alpha W_{j}}{N_{j}} .
$$

Note that the second side constraint may hold with equality for faculty $A$.

As a first step towards characterizing the subgame-perfect equilibrium we analyze the minimal status requirements of the respective faculties as a function of the wages offered. Therefore, we use the indifference condition, which faculty $A$ must ensure that it holds, and solve $\Delta_{j}^{m_{j}}=0$ for $s^{C}$. This yields:

$$
\begin{aligned}
& s_{A, \min }\left(W_{B}\right)=\frac{\sum_{A} s^{i}-s^{m_{A}}}{N_{A}-1}-\alpha \cdot\left(\frac{\sum_{A} s^{i}}{N_{A}}-\frac{\sum_{B} s^{i}}{N_{B}}\right)+\alpha W_{B}, \\
& s_{B, \min }\left(W_{B}\right)=\frac{\sum_{B} s^{i}-s^{m_{B}}}{N_{B}-1}+\alpha W_{B} .
\end{aligned}
$$

For presentational simplicity, we rewrite (12) and (13) as: ${ }^{8}$

$$
\begin{aligned}
& s_{A, \min }\left(W_{B}\right)=\hat{s}_{A}^{m_{A}}-\alpha\left(\hat{s}_{A}-\hat{s}_{B}\right)+\alpha W_{B}, \\
& s_{B, \min }\left(W_{B}\right)=\hat{s}_{B}^{m_{B}}+\alpha W_{B} .
\end{aligned}
$$

\footnotetext{
${ }^{8}$ Recall that $\hat{s}_{j}^{m}$ refers to the status utility enjoyed by the median member of faculty $j$, whereas $\hat{s}_{j}$ refers to the average status of faculty $j$, which coincides with the status utility enjoyed by an entrant to $j$.
} 
Since $\Delta_{j}^{m_{j}}$ is strictly increasing in $s^{C}$, this implies that faculty $j$ will not make any acceptable offer to candidates with $s^{C}<s_{j, \min }$. Comparing the minimal status position determined by the two faculties we find:

$$
s_{A, \min }-s_{B, \min }=\hat{s}_{A}^{m_{A}}-\hat{s}_{B}^{m_{B}}+\alpha\left(\hat{s}_{B}-\hat{s}_{A}\right) .
$$

Note that (16) is independent of the wages offered by the faculties. This fact, which stems from the assumed symmetry across faculties with respect to $\alpha$ and the additively separable utility function, allows for independent analysis of the faculties' choices of minimal status requirement and wage offered. The first difference on the RHS of (16) reflects the (positive) difference in the "willingness-to-accept" a certain candidate associated with the impact on the ex ante utility enjoyed by the median member in faculty $A$ compared to his counterpart in faculty $B$. In contrast, the second term in (16) reflects the "necessity-to-pay" for a new member by faculty $A$ relative to the one in faculty $B$. This difference is negative because every new entrant gains relatively more when entering faculty $A$ than faculty $B$, given the higher average status level in faculty $A$. This implies that the new member is willing to accept a lower salary offer from faculty $A$ than from faculty $B$. The sign of the RHS of (16) depends, thus, on the relative impact of the two effects.

Proposition 1 (Entry thresholds) Let $z \equiv \min \left\{1, \frac{\hat{s}_{A}^{m} \hat{s}_{A} \hat{s}_{B}^{m}}{\hat{s}_{A}-\hat{s}_{B}}\right\}$. Then,

(i) Given that substitution between different budgetary issues is difficult $(\alpha<z)$, the required minimal status position of faculty $B$ is strictly lower than the one of faculty $A$ (that is, $s_{A, \min }>s_{B, \min } \forall W_{B}$ ).

(ii) With budgetary substitution being rather simple $(\alpha \geq z)$, faculty $B$ is more restrictive than faculty $A$ (that is, $s_{A, \min } \leq s_{B, \min } \forall W_{B}$ ).

The second part of Proposition 1 emerges if and only if the status distributions of the two faculties are skewed differently. If the difference between median and average status level in both faculties is the same (or smaller in $A$ than in $B$ ), then $z=1$, which implies (because $\alpha$ is assumed to be strictly smaller than one) that only case (i) is feasible. With all other status distributions, the second case becomes feasible but will emerge only if $\alpha$ is sufficiently large. This occurs only if an increase in the financial resources of the faculty are nearly as valuable for incumbent faculty members as an increase in own salary. Given that the main focus of our analysis is on faculties in which the social interaction among members leads to improved research outcomes, this is not the point of interest in the current paper. Therefore, we concentrate our analysis on case (i) of Proposition 1. ${ }^{9}$

Before we characterize the subgame-perfect equilibrium, let us define the following wage levels. The minimal salary a candidate might accept from faculty $B$ is given by:

$$
W_{B}^{E} \equiv R-\hat{s}_{B}
$$

\footnotetext{
${ }^{9}$ Note that the implications of the second case for the analysis in this section are quite straightforward (the results in all other sections remain unchanged): If faculty $A$ is less restrictive than faculty $B$ ( $s_{A, \min } \leq$ $\left.s_{B, \text { min }}\right)$, a candidate will enter faculty $A$ independent of his own status ${ }^{C}$ because $A$ is, for any given wage level $W_{B}$, more attractive for the candidate and more willing to accept new members than faculty $B$. Hence, if $\alpha \geq z$, no candidate will join faculty $B$.
} 
Fig. 1 Stratified segmentation of candidates in faculties $A$ and $B$

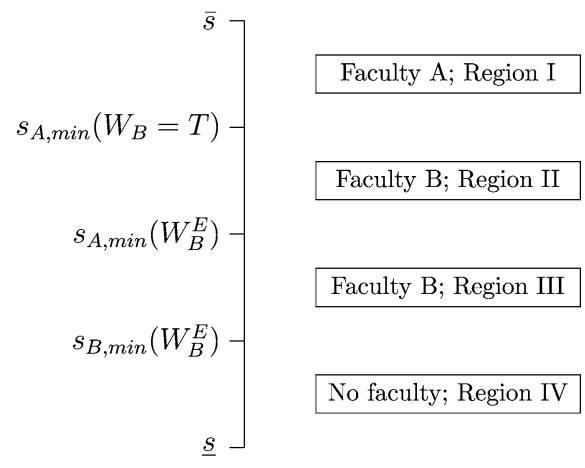

The minimal competitive salary of faculty $B$, still allowing faculty $B$ to attract the new candidate, is given by:

$$
W_{B}^{+} \equiv\left(\hat{s}_{A}-\hat{s}_{B}\right)+\frac{s^{C}}{\alpha}-\frac{\hat{s}_{A}^{m_{A}}}{\alpha}+\epsilon,
$$

where $\epsilon$ is the smallest feasible monetary unit. Furthermore, we define the minimal salary offered by faculty $A$ that lets the candidate's IC (indifference condition) hold if faculty $B$ offers the candidate its entire hiring budget, $T$, as wage:

$$
\tilde{W}_{A} \equiv T-\left(\hat{s}_{A}-\hat{s}_{B}\right) .
$$

We prove in the Appendix A:

Proposition 2 (Equilibrium with majority voting) Assume that $\alpha<\frac{\hat{s}_{j}^{m_{j}}-\underline{s}}{\hat{s}_{j}} \forall j$. The subgameperfect equilibrium can be characterized as follows:

(i) Region IV: A candidate with very low status, $s^{C}<s_{B \text {, min }}\left(W_{B}^{E}\right)$, does not get an acceptable offer from either faculty.

(ii) Region III: A candidate with low status level, $s^{C} \in\left[s_{B, \min }\left(W_{B}^{E}\right), s_{A, \min }\left(W_{B}^{E}\right)\right)$, gets an acceptable offer only from faculty $B$, which he accepts. $W_{B}=W_{B}^{E}$.

(iii) Region II: A candidate with medium status level, $s^{C} \in\left[s_{A, \min }\left(W_{B}^{E}\right), s_{A, \min }\left(W_{B}=T\right)\right)$, receives acceptable offers from both faculties. He joins faculty $B$ for a wage $W_{B}=W_{B}^{+}$.

(iv) Region I: A candidate with high status level, $s^{C} \geq s_{A \text {, min }}\left(W_{B}=T\right)$, receives acceptable offers from both faculties. He joins faculty A for a wage of $W_{A}=\tilde{W}_{A}$.

(v) The faculty losing the competition for the candidate in a region offers a wage as competitive as possible (such that $\Delta_{j}^{m_{j}}=0$ for that faculty's median member).

If $\alpha \geq \frac{\hat{s}_{B}^{m}{ }^{B}-s}{\hat{s}_{B}}$, region IV does not exist and all candidates are accepted by some faculty. If $\alpha \geq \frac{\hat{s}_{A}^{m} A-s}{\hat{s}_{A}}$, region III also does not exist. As these two cases do not affect our main predictions, namely that some candidates enter faculty $A$ for this wage whereas others enter faculty $B$ for that wage, we will analyze the full spectrum of regions as characterized in Proposition 2.

Figures 1 (delineating the allocation of entrants to faculties) and 2 (plotting the wage paid by the "winning" faculty) illustrate Proposition 2. 
Fig. 2 Wage paid by the "winning" faculty

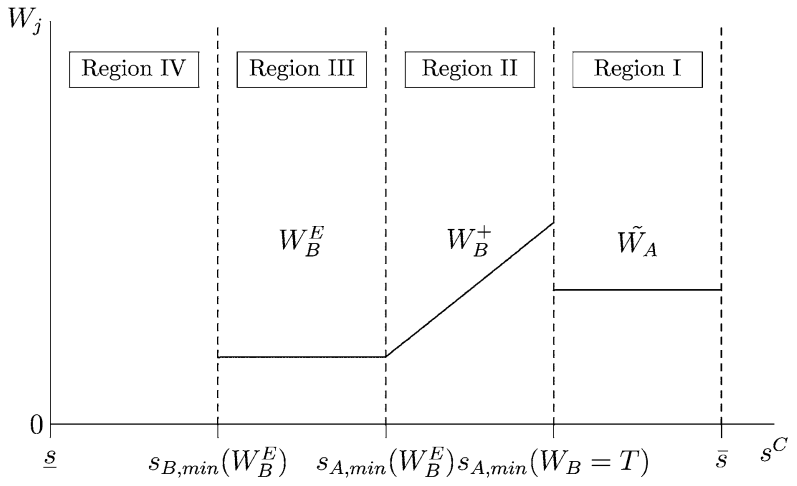

In region IV, there is no wage that satisfies a candidate's participation constraint and produces a nonnegative utility differential for the median member $m_{B}$ (let alone $m_{A}$ ). Thus, candidates in this region stick with their outside option. For candidates in region III, faculty $B$ is protected from competition by faculty $A$ as the median voter of faculty $A$ would want to compete with for the candidate only if the wage offer is quite low. This would, however, violate $\left(P C_{A}\right)$. In region $\mathrm{I}$, on the other hand, faculty $A$ is protected from intense competition. Because of its budget constraint, $T$, faculty $B$ is not able to offer a high status candidate a package of an attractive research environment and a wage that exceeds faculty $A$ 's. In region II, competition for the candidate is most intense: neither faculty is protected from competitive bids by the other faculty. Due to the impact of $W_{B}$ on $s_{A, \min }$, however, faculty $B$ can offer a wage, $W_{B}^{+}$, that is sufficiently attractive for the candidate such that an equally attractive wage offer from faculty $A$ would turn the utility differential of $A$ 's median member slightly negative. Consequently, the candidate will enter faculty $B$.

The intuition of Proposition 2.(v) is that the "losing" faculty $j$ neither has an incentive to bid a higher wage than the most competitive wage (as it would violate $s_{j, \min }$ and make $\Delta_{j}^{m_{j}}<0$ ) nor to propose a lower wage (this would make membership in faculty $j$ even less attractive for the candidate and would not change $m_{j}$ 's surplus of zero). Given this strategy of the losing faculty, the "winning" faculty's best response, according to the arguments above, is to offer $W_{B}^{E}, W_{B}^{+}$and $\tilde{W}_{A}$ in the respective regions III to I.

As a direct consequence of Proposition 2 we have the following:

Corollary 1 (Wage levels) In equilibrium the highest ranking candidates (in region I) receive lower wages than some candidates with relatively lower status (the best in region II).

In other words, top ranking researchers join a top ranking faculty for comparatively low remuneration, whereas some researchers with lower status join lower ranking faculties for a comparatively high salary. Figure 2 illustrates this idea. ${ }^{10}$

\subsection{The allocation of surplus}

To enable us to study the effects of competition for candidates on the utility of all individuals involved, we follow a two-step procedure. First, we analyze how the surplus created by entry

\footnotetext{
${ }^{10}$ Note that in Fig. 2 we plotted the equilibrium wages of the winning faculty for $\hat{s}_{A}<2 \hat{s}_{B}+T-R$. If this inequality did not hold, we would have $W_{B}^{E} \geq \tilde{W}_{A}$. In region II, $W_{B}^{+}$would adjust accordingly, starting from the low level of $W_{B}^{E}$ at $s_{A, \min }\left(W_{B}^{E}\right)$ and increasing linearly to a value of $T$ at $s_{A, \min }\left(W_{B}=T\right)$.
} 
of a candidate is divided among the three key players: the median members of faculties $A$ and $B$, and the candidate. Second, we analyze the distribution of surplus within the faculty that hires the candidate, the "winning" faculty.

Equation (1) states that, without the entry of a candidate, the median member of faculty $j$ obtains a utility level of $\hat{s}_{j}^{m_{j}}+\alpha T / N_{j}$. The candidate gets his outside option, $R$. Conditions (5) and (6) capture the utility differentials of the three key players conditional on the event that the candidate enters a faculty, compared to taking their outside options. Henceforth, we will use the terms utility differential and surplus exchangeably.

By definition, the utility differential of the "losing" median member, who does not get a new colleague, is $\Delta_{j}^{m_{j}}=0$. The utility differential of the winning median member, whose faculty hires the candidate, is given by (6), which depends on the equilibrium wage. We prove the following Lemma in the Appendix A.2.

Lemma 2 (Surplus division between the key players) (i): In region IV, the surplus of all three key players is zero. (ii): In region III, only the median member of faculty B enjoys positive surplus, which is increasing in the status of the candidate. (iii): In region II, only the candidate gains positive surplus, which is increasing in his own status. (iv): In region I, both the candidate and the median member of faculty A receive positive surplus, given that $R$ is sufficiently low, but only the median member's surplus increases in the candidate's status.

Lemma 2 reflects the varying degree of competition between the two faculties in the respective regions, allowing faculty $B(A)$ to achieve a positive surplus in region III (I). The fact that the two faculties are protected from competition in these two regions implies that they can capture the entire increase in total surplus that arises if candidates with more status are hired. In region II, in which competition is intense, it is the candidate who can collect the entire surplus. In addition, Lemma 2 shows that, depending on the status of the candidate, each key player can generate a positive surplus from entry at some point.

Now we turn to the question, how is the surplus that is generated by entry of a candidate distributed within the winning faculty? To answer this question we first need to define the aggregate surplus of the winning faculty. It is, following (6):

$$
\Delta_{j} \equiv \sum_{k} \Delta_{j}^{k}=s^{C}-\hat{s}_{j}-\alpha W_{j}
$$

In the next Lemma, which is proven in the Appendix A.3, we state the surplus of the winning faculty in each region. By definition, the surplus of the losing faculty is zero. The surplus of the candidate is given by Lemma 2. In region IV, neither faculty wins.

Lemma 3 (Joint surplus of incumbents of winning faculty) At the lower bound of a region, the surplus of the winning faculty $(j)$ is:

$$
\hat{s}_{j}^{m_{j}}-\hat{s}_{j}
$$

The joint surplus of the incumbents of the winning faculty increases with $s^{C}$ in region III ( for faculty $B$ ) and in region I (for faculty $A$ ), whereas it stays constant in $s^{C}$ in region II.

Lemmas 2 and 3 put us in a situation where we can compare the surplus of the median member of the winning faculty with the joint surplus of all incumbent faculty members. We 
rule out that wages for incumbent faculty members can be adjusted individually after entry of a candidate to correct for different status levels. ${ }^{11}$

At the lower bound of each region, the median member of the winning faculty $j$ receives zero surplus, while the aggregate surplus of his faculty is $\Delta_{j}=\hat{s}_{j}^{m_{j}}-\hat{s}_{j}$. If this expression is positive, it implies that a candidate with a status level marginally below the lower bound of the region is not admitted to the faculty at all or, at least, not admitted for the wage paid in the equilibrium of the region (see Proposition 2). However, from the aggregate member perspective, it would have been beneficial to admit the candidate for the equilibrium wage, that is, it would have been beneficial to lower $s_{j, \min }$. It follows that, in this case, the median member of the winning faculty implemented a hiring policy that is too restrictive.

In turn, if $\Delta_{j}=\hat{s}_{j}^{m_{j}}-\hat{s}_{j}$ is negative, it implies that a candidate with a status level equal to the lower bound of the region is admitted to the faculty for the equilibrium wage of that region. However, from the aggregate perspective, it would have been beneficial not to admit the candidate for the equilibrium wage, that is, it would have been beneficial to increase $s_{j, \min }$. Hence, in this case, the median member of the winning faculty implemented a hiring policy that is too liberal. Based on the above discussion we can state the following Proposition without a formal proof.

Proposition 3 (Entry and joint surplus of the winning faculty) (i). If $\hat{s}_{j}^{m_{j}}>(<) \hat{s}_{j}$, the amount of entry in faculty $j$ is suboptimally low (high) from the perspective of the joint surplus of incumbent members of faculty $j$. (ii). For $\hat{s}_{j}^{m_{j}}=\hat{s}_{j}$, the minimal status threshold is optimal.

The intuition of Proposition 3 is straightforward if we realize that $\hat{s}_{j}^{m_{j}}>(<) \hat{s}_{j}$ implies that the median voter is located to the left (right) of the center of the distribution implying that the median voter is too restrictive (liberal) when deciding on entry. Hence, entry is suboptimally low (high). If the median voter is located precisely at the center, this suboptimality is avoided.

Therefore, our analysis suggests that majority voting is an approximation of an optimal decision-making rule for faculties in which the status distribution is rather symmetric.

\section{Robustness}

We focus in this section on what we consider the main assumptions of our model and discuss the implications of relaxing them.

Faculty size The main mechanism in our analysis hinges on the fact that faculty members with higher status gain relatively more from a new member than members with a lower status. This mechanism arises since all incumbent faculty members get the same gross benefit from the interaction with a new member but they bear different opportunity costs: lower-tier members have to share opportunities of interacting with higher status members with another colleague (dilution effect), whereas faculty "stars" give up less. They benefit more from the budget residual, that is from the monetary contribution left over after admitting a new member. The dilution effect is most prevalent with a rather small number of faculty members

\footnotetext{
${ }^{11}$ This assumption is in line with the common practice in most academic faculties. The opposite assumption would obviously come at high transaction costs because the dean would have to solve $N_{j}$ bargaining games simultaneously - after every change in faculty composition.
} 
and is mitigated as faculty size increases. Therefore, we consider our main mechanism to be robust as long as we do not study faculties that are very large.

Nonrivalry and different marginal valuations of status Our mechanism rests on two assumptions in the baseline model. (i) Status is a rival good. (ii) Each member of faculty $j$ assigns the same weight to the utility derived from the average status of the other faculty members.

In Appendix B.1, we show that our key results, which are based on Lemma 1 and Proposition 1, hold both for status being a rival or a partially nonrival good. Relaxing the second assumption, case (ii), is more complex. We show in Appendix B.2 that Lemma 1 and Proposition 1 hold qualitatively if the marginal status utility that a player $k$ gets does neither increase nor decrease too steeply in his own status rank, $s^{k}$. We show that, if the weight on status utility increases too steeply in status rank, faculty $A$ does not leave any candidates to faculty $B$ that faculty $B$ wants to accept (that is, $s_{A, \min } \leq s_{B, \min }$ ). If the weight on status utility decreases too steeply in status rank, $s_{A, \min }-s_{B, \min }>0$, but grows so large that faculty $A$ will not make a competitive offer to candidates anymore because it values candidates' status much less than faculty $B$. In general, given that we observe stratification of faculties and candidates empirically and do not observe that only high ranking or only low ranking faculties capture the entire job market for academics, we consider our model using equal valuations of status across faculty members as a better approximation of reality than a model with rather extreme assumptions of status utility depending on members' own status.

Positive assortative matching In our baseline model, we assumed that collaboration among faculty members is uniformly distributed, in expectation. In Appendix A.3, we study the effects of an alternative, yet equally plausible matching pattern. We consider the case where matches between pairs of faculty members are "localized", that is that high (low) status members are more likely to interact with other high (low) status members. This implies that matching between faculty members is positively assortative. Specifically, we capture positive assortative matching by assuming that the utility from membership on a faculty follows a binomial distribution, where the probability of being matched with high status members increases in a member's own status rank.

We show that Lemma 1 is unaffected by this assumption: the minimal status requirement for new faculty hires still decreases strictly and monotonically in rank. Proposition 1, which lays the foundation for our main result, Proposition 2, is even strengthened as there are cases where the relation of $\alpha$ and $z$ is irrelevant for Proposition 1.(i) to hold.

Asymmetric utility functions between members and candidates We assumed symmetry of the utility functions between incumbent faculty members, on the one side, and candidates, on the other. If, for instance, the candidate values status less than money (as compared to incumbent faculty members), the competitive advantage of faculty $A$ decreases. The difference in wages becomes more important. This effect becomes most obvious if the candidate is interested only in wage offers. A new entrant with high status is relatively more attractive for faculty $B$ than for faculty $A$ (since the effect on average status is more pronounced for faculty $B$ ). Hence, faculty $B$ is able and willing to attract high status candidates. A potentially relevant application of this might be if highly reputable professors prefer second-tier universities, thereby making more money than by joining a top-university. In the absence of compelling reasons to expect systematic differences in preferences, we believe that the assumption of symmetric preferences is the most reasonable. 
Relaxing budget constraints The driving force of our analysis is the trade-off between utility from status and from money. As long as there is any budget constraint for the two faculties (allowing for the non-existence of budget constraints obviously is implausible), the quality of our results remains intact. If we start from our initial assumption and relax budget constraints, faculty $B$ can compete more fiercely with faculty $A$ in monetary terms, that is, it can offer a higher wage. This change leads to a shrinking of region I but it leaves all other results intact.

Repeated interaction If we extend the one-shot game in our baseline model to a repeated game setting, the results will depend on the shape of the ex ante and the ex post (after entry took place once) frequency distributions of members in both faculties. What is important for our analysis is the insight that our results are robust in each stage of the game as long as it complies with our parameter setting in Proposition 1.(i).

Many competing faculties Are our results robust to an extension to many faculties? Consider the case in which the status of a new candidate is drawn from a distribution over $[\underline{x}, \bar{x}]$, where $\underline{x} \ll \underline{s}$ and $\bar{x} \gg \bar{s}$. Assume that, out of many vertically stratified faculties, we focus on faculties $Z, A$, and $B$, where faculty $Z$ has a higher average status than faculty $A$, which is in turn of higher average status than $B$. Assumption (3) holds here as well. When determining the entry regions, as in Proposition 2, the threshold between faculties $A$ and $B$ remains $s_{A, \min }\left(W_{B}=T\right)$. However, in this setting faculty $A$ also faces competition from the higher status faculty $Z$. Taking the intuition of Proposition 1 into account, faculty $A$ has incentives to offer a higher wage than faculty $Z$ to any new candidate, until its budget constraint becomes binding, at $T$. We conclude that we can expect entry in faculty $A$ for all new candidates with $s^{C} \in\left[s_{A, \min }\left(W_{B}=T\right), s_{Z, \min }\left(W_{A}=T\right)\right]$, where $s_{A, \min }\left(W_{B}=T\right)=\hat{s}_{A}^{m_{A}}-\alpha\left(\hat{s}_{A}-\hat{s}_{B}\right)+\alpha T$ and $s_{Z, \min }\left(W_{B}=T\right)=\hat{s}_{Z}^{m_{Z}}-\alpha\left(\hat{s}_{Z}-\hat{s}_{A}\right)+\alpha T$. This interval is non-empty for:

$$
\alpha<\frac{\hat{s}_{Z}^{m_{Z}}-\hat{s}_{A}^{m_{A}}}{\hat{s}_{Z}+\hat{s}_{B}-2 \hat{s}_{A}} .
$$

Then each faculty has a positive probability of gaining the candidate as a new member in equilibrium. Wages are determined according to the result of region II, the most competitive region in our basic setting.

\section{Conclusion}

In this paper, we have studied the competition among academic faculties for job candidates, in which the incumbent members have authority over recruitment of new members. The main-and novel-assumption of our model is that faculties are status organizations, wherein a member's utility depends on the average research capability of his fellow members. Thus, utility from faculty membership differs among all members, unless they have the same status.

We have shown that high status faculty members set a lower status threshold for job candidates to enter the faculty, that is they are more liberal than low status faculty members. Our main result is that high status candidates join the higher ranking faculty but for a medium wage, whereas lower ranking candidates join the lower ranking faculty. Interestingly, the best candidates joining the lower ranking faculty receive higher wages than the candidates joining the better faculty. These results are testable empirically. 
Moreover, we have shown that a majority voting rule, which this study focuses on, can create too much or too little entry, from the aggregate faculty members' perspective. These inefficiencies are avoided in faculties where the status of all members is distributed symmetrically around the mean.

Our model applies to a wide range of potential applications beyond our particular example of academic institutions. The defining characteristics are, first, that members' utility depends on the status positions of their fellow members. Second, the organizations must be member-owned, that is the incumbent members possess the decision rights over the entry of future members. Third, money and status are not perfect substitutes.

In the baseline model, we have focused on academic faculties, implying that new entrants receive wages rather than pay entrance fees. However, our analysis is not restricted to this case. If we define $W_{j} \leq 0$ to be a fee paid by a candidate to an organization, our full analysis also applies. In this case we could even lift two restrictions of the baseline model and allow for positive and negative $T \mathrm{~s}$ (total recruitment budgets) as well as positive and negative $R \mathrm{~s}$ (the returns available to candidates in their outside options). The highest ranking candidates would still join the better organization but pay a larger entry fee than some lower ranking candidates (cf. Corollary 1). All other results hold accordingly.

This generalizability allows for a transfer of our findings to a wide variety of organizations and institutions, including country clubs, golf clubs, and student clubs. It also applies to international economic institutions, such as the European Currency Union or the World Trade Organization, and to mutual fund insurances.

There are a number of avenues for future research. First, we have assumed in this first model of its kind that status is a unidimensional vertically differentiable variable. Relaxing this assumption by allowing both faculties and candidates also to position themselves on a horizontal dimension may trigger interesting strategic interactions. A more ambitious extension would relax the assumption of majority voting and endogenize the governance structure of academic faculties. For this purpose it would be necessary to define (and justify) the objective functions of all players who could have property rights with respect to faculty governance, including purely administrative (nonacademic) deans and university executive boards. Related to this approach, analyzing the implications of competition among investor-owned clubs (such as some professional sports clubs) would be particularly interesting. Here, too, it would be crucial to start by defining the objective functions of the members of such organizations.

Acknowledgements We owe thanks to Matthias Blonski, Patrick Herbst, Christian Laux, Scott Masten, Alfons Weichenrieder, seminar participants in Frankfurt and Konstanz, and participants of the EEA annual meeting in Vienna, the EARIE conference in Amsterdam, the Verein für Socialpolitik Jahrestagung in Bayreuth, and the ISNIE conference in Boulder, Colorado. We are also very grateful to two anonymous referees for valuable comments and suggestions on a previous version of the paper.

Open Access This article is distributed under the terms of the Creative Commons Attribution Noncommercial License which permits any noncommercial use, distribution, and reproduction in any medium, provided the original author(s) and source are credited.

\section{Appendix A: Proofs}

\section{A.1 Proof of Proposition 2}

(i): Because of Proposition 1.(i), the cut-off status level below which candidates do not get an acceptable offer from any faculty is determined by faculty $B . W_{B}^{E}$ denotes the salary 
for which $\left(P C_{B}\right)$ of the entrant just holds with equality. As $s_{B, \min }$ is increasing in $W_{B}$, $s_{B, \min }\left(W_{B}^{E}\right)$ is the lowest status level at which faculty $B$ makes an offer that meets $\left(P C_{B}\right)$.

(ii): By definition of $s_{A, \min }$, in the range $s^{C} \in\left[s_{B, \min }\left(W_{B}^{E}\right), s_{A, \min }\left(W_{B}^{E}\right)\right)$ faculty $A$ is not able to make a membership offer to the candidate that satisfies both parties (independently of faculty $B$ 's behavior). Therefore, faculty $B$ is able to exploit the candidate completely, which means setting $W_{B}=W_{B}^{E}$.

(iii): For $s^{C} \geq s_{A, \min }\left(W_{B}^{E}\right)$, demanding $W_{B}=W_{B}^{E}$ has the consequence that faculty $A$ has an incentive to match the offer of faculty $B$. The candidate would then join faculty $A$. In the range $s^{C} \in\left[s_{A, \min }\left(W_{B}^{E}\right), s_{A, \min }\left(W_{B}=T\right)\right)$, however, faculty $B$ can make sure that $s_{A, \min }\left(W_{B}\right)>s^{C}>s_{B, \min }\left(W_{B}\right)$. Thus, faculty $A$ has no incentive to offer the candidate entry with a salary that would both meet $\left(P C_{A}\right)$ and make him prefer membership in faculty $A$ over faculty $B$. Because of the second part of this inequality, faculty $B$ still has this incentive, though. As an increase in wages increases $s_{A, \min }$ and $s_{B, \min }$ by the same factor, $\alpha$ (see (14) and (15)), $B$ can sustain this behavior throughout region II. By using (14) we can find $W_{B}^{+}$ as defined above, whereby $\epsilon$ denotes a very small number and $\left(\partial W_{B}^{+}\right) /\left(\partial s^{C}\right)>0$. Hence, an entrant with $s^{C}$ very close to $s_{A, \min }\left(W_{B}=T\right)$ receives almost the maximum wage that faculty $B$ can pay given its budget.

(iv): Because of the budget constraint of faculty $B, W_{B}$ cannot be increased indefinitely. Hence, faculty $B$ has no way of preventing faculty $A$ from making offers to candidates with $s^{C} \geq s_{A, \min }\left(W_{B}=T\right)$ that are mutually beneficial. Faculty $A$ can let the indifference condition (and the participation constraint) of the candidate hold. Comparing (7) and (9) reveals that, from the point of view of faculty $A$, (9) is (weakly) more restrictive if $W_{B} \geq$ $R-\hat{s}_{B}$, that is if $\left(P C_{B}\right)$ holds. This condition holds $\forall s^{C} \geq s_{B, \min }\left(W_{B}^{E}\right)$, that is in regions III, II, and I. This result implies that, if faculty $A$ offers a wage in region I for which the indifference condition holds, the participation constraint holds, too. Thus, faculty $A$ offers a wage level which is equal to the difference of the average status levels between the two faculties from the point of view of the entrant. This consideration yields $W_{A}=\tilde{W}_{A}$.

(v): The faculty losing the competition for the candidate (both faculties in region IV, faculty $A$ in regions III and II, faculty $B$ in region I) gets an expected surplus of zero, no matter which strategy it employs. If it plays the most competitive strategy and offers a $\left(s_{j, \min }, W_{j}\right)$-combination such that $\Delta_{j}^{m_{j}}=0$, it still has no incentives to deviate. However, it ensures that the actions characterized in parts (i)-(iv) of the Proposition are incentivecompatible.

\section{A.2 Proof of Lemma 2}

The surplus of the key players is captured by $\Delta_{A}^{m_{A}}, \Delta_{B}^{m_{B}}$, and $\Delta_{C}$.

(i). In region IV, no entry takes place. Hence, there we have $\Delta_{A}^{m_{A}}=\Delta_{B}^{m_{B}}=\Delta_{C}=0$.

(ii). In region III, the candidate chooses to join faculty $B$ but will not get any surplus from entry as $W_{B}^{E}$ just pays him his reservation value: $\Delta_{C}=\Delta_{A}^{m_{A}}=0$. In contrast, $\Delta_{B}^{m_{B}}\left(W_{B}^{E}\right)=$ $\frac{1}{N_{B}}\left(s^{C}-\hat{s}_{B}^{m_{B}}-\alpha\left(R-\hat{s}_{B}\right)\right)$. By definition of $s_{B, \min }\left(W_{B}^{E}\right)$, this expression is zero at its lower bound. It is increasing in $s^{C}$.

(iii). In region II, the candidate also joins faculty $B$. Hence, $\Delta_{A}^{m_{A}}=0$. Substituting $W_{B}^{+}$in (6) yields $\Delta_{B}^{m_{B}}\left(W_{B}^{+}\right)=\frac{1}{N_{B}}\left(s^{C}-\hat{s}_{B}^{m_{B}}-\alpha\left(\hat{s}_{A}-\hat{s}_{B}+\frac{{ }^{C} C}{\alpha}-\frac{\hat{s}_{A}^{m_{A}}}{\alpha}+\epsilon\right)\right)$. Note that $s^{C}$ cancels from this expression. By definition, $\Delta_{B}^{m_{B}}\left(W_{B}^{+}\right)=0$ at its lower bound, which does not change over the course of region II because it is constant in $s^{C}$. In contrast, the candidate benefits from higher own status value: $\Delta_{C}=\frac{{ }_{s^{C}-\hat{s}_{A} m_{A}}}{\alpha}+\hat{s}_{A}+\epsilon-R$. This differential is zero at its lower bound, $s_{A, \min }\left(W_{B}^{E}\right)$, and grows linearly with increasing $s^{C}$. 
(iv). In region I, the candidate joins faculty $A$. Thus, $\Delta_{B}^{m_{B}}=0$. Substituting $\tilde{W}_{A}$ in (6) yields $\Delta_{A}^{m_{A}}=\frac{1}{N_{A}}\left(s^{C}-\hat{s}_{A}^{m_{A}}-\alpha\left(T-\left(\hat{s}_{A}-\hat{s}_{B}\right)\right)\right)$. This expression is zero at its lower bound, $s_{A, \min }\left(W_{B}=T\right)$, and increases in $s^{C}$. The candidate receives a utility differential of $\Delta_{C}=$ $T+\hat{s}_{B}-R$, which is positive for sufficiently low $R$ and constant in $s^{C}$.

\section{A.3 Proof of Lemma 3}

In region III, $\Delta_{B}=s^{C}-(1-\alpha) \hat{s}_{B}-\alpha R$, which is increasing in $s^{C}$. By definition of $s_{B, \min }\left(W_{B}^{E}\right)$, the status level of an admitted candidate in region III is at least $s^{C}=\hat{s}_{B}^{m_{B}}+$ $\alpha\left(R-\hat{s}_{B}\right)$. Substituting this into $\Delta_{B}$ yields $\Delta_{B}\left(s^{C}=s_{B, \min }\left(W_{B}^{E}\right)\right)=\hat{s}_{B}^{m_{B}}-\hat{s}_{B}$ in the case of the lowest ranked admitted candidate. Depending on the ex ante frequency distribution of status in faculty $B$, this can be positive or negative.

In region II, substituting $W_{B}^{+}$in (17) yields $\Delta_{B}=\hat{s}_{A}^{m_{A}}-\alpha \hat{s}_{A}-(1-\alpha) \hat{s}_{B}-\alpha \epsilon$. In case of the lowest ranked candidate admitted in region II, this yields $\Delta_{B}\left(s^{C}=s_{B, \min }\left(W_{B}^{+}\right)\right)=$ $\hat{s}_{B}^{m_{B}}-\hat{s}_{B}$. Just as in region III, this can be positive or negative. In contrast to region III, $\Delta_{B}$ is not increasing in $s^{C}$.

In region I, substituting $\tilde{W}_{A}$ in (17) yields $\Delta_{A}=s^{C}-(1-\alpha) \hat{s}_{A}-\alpha \hat{s}_{B}-\alpha T$, which is increasing in $s^{C}$. For the lowest ranked candidate admitted in region I, this yields $\Delta_{A}\left(s^{C}=\right.$ $\left.s_{A, \min }\left(W_{B}=T\right)\right)=\hat{s}_{A}^{m_{A}}-\hat{s}_{A}$. Depending on the ex ante frequency distribution of status in faculty $A$, this can be positive or negative.

\section{Appendix B: Alternative model specifications}

\section{B.1 Nonrivalry of status}

In our baseline model, the rivalry of status is captured by using the average status of the faculty as an argument in the utility function. On the other hand, to model status as a completely nonrival good the sum of the status levels of all other faculty members should be used. By this approach, a generalization of (2) is given by:

$$
\hat{s}_{j}^{k}=\frac{\sum_{j} s^{j}-s^{k}}{\left(N_{j}-1\right)^{\delta}}
$$

with $\delta \in[0,1]$ denoting the degree of rivalry in status. The larger is $\delta$, the larger the degree of rivalry. With $\delta$ close to zero, status is almost completely a nonrival good. With $\delta=1$ we are back to our baseline model. This specification turns our critical equation (6) into:

$$
\Delta_{j}^{k}=\frac{s^{C}}{N_{j}^{\delta}}-\frac{\left(\sum_{j} s^{i}-s^{k}\right)\left(N_{j}^{\delta}-\left(N_{j}-1\right)^{\delta}\right)}{\left(N_{j}-1\right)^{\delta} N_{j}^{\delta}}-\alpha \frac{W_{j}}{N_{j}} .
$$

Note that the (dis)utility of each incumbent faculty member from paying the new member a wage is independent of $\delta$ because money is a completely rival good. Moreover, note that, $N_{j}^{\delta}>\left(N_{j}-1\right)^{\delta}, \forall \delta>0$; hence $\partial \Delta_{j}^{k} / \partial s^{k}>0$ (still assuming that $\sum_{j} s^{i}$ is unaffected when considering the change in $s^{k}$ ). In contrast, for $\delta=0$, that is, if status is a completely nonrival good, $\Delta_{j}^{k}$ reduces to $s^{C}-\alpha \frac{W_{j}}{N_{j}}$. This case does not depend on the status distribution across faculties. In other words, if status was a completely nonrival good and budget constraints were not binding, the larger of the two faculties (with $N_{j}>N_{q \neq j}$ ) would offer a higher wage to all candidates independent of their status level. The market would be at a tipping 
point. Given that we do not observe such a radical outcome in practice, we proceed in our analysis for $\delta>0$, that is, if status has at least some rival component. This implies, together with $\partial \Delta_{j}^{k} / \partial s^{C}>0, \forall \delta$ in (B.1), that Lemma 1 is robust.

Proceeding with the analysis of this model specification, it is straightforward to see that (16), comparing the minimal status positions set by the two faculties, remains structurally unchanged. It follows that Proposition 1 (and all subsequent results) also remains qualitatively unchanged if we allow for status to be a partially nonrival good. ${ }^{12}$

\section{B.2 Different valuation of status across faculty members}

In this appendix, we allow for different valuations of fellow faculty members' status across faculty members. Obviously, this could go either way. Depending on individual preferences as well as types of interactions, high status faculty members might benefit more (less) from a high average status level of the respective faculty as compared to faculty members with low status. In order to allow for all feasible types of varying valuations of status across different faculty members we use the following general utility function, related to (1):

$$
u_{j}^{k}=\left(s^{k}\right)^{\beta} \hat{s}_{j}^{k}+\alpha\left(\frac{T-W_{j}}{N_{j}}\right)
$$

where $\left(s^{k}\right)^{\beta}$ measures how the status utility depends on member $k$ 's own status. $\beta \in \mathbb{R}$ specifies the marginal utility that $k$ gets from collaboration. ${ }^{13}$ For $\beta>0(\beta<0)$, the marginal status utility is higher (lower) for highly ranked members than for lowly ranked members. For $\beta=0$, that is, the in-between case, the marginal status utility from entry is independent of $k$ 's own rank, which is the case in our baseline model.

Using this specification of the utility function, (6) turns into:

$$
\Delta_{j}^{k}=\frac{1}{N_{j}}\left(\left(s^{k}\right)^{\beta}\left(s^{C}+\frac{s^{k}-\sum_{j} s^{i}}{\left(N_{j}-1\right)}\right)-\alpha W_{j}\right) .
$$

We find that $\partial \Delta_{j}^{k} / \partial s^{C}>0$ (given that $\sum_{j} s^{i}$ is unaffected by the change in $s^{k}$ ) and

$$
\operatorname{sign} \frac{\partial \Delta_{j}^{k}}{\partial s^{k}}=\operatorname{sign}\left(\beta s^{C}+\frac{(1+\beta) s^{k}-\beta \sum_{j} s^{i}}{N_{j}-1}\right) .
$$

Hence, $\beta$ not being too large (in absolute terms) is a sufficient condition for Lemma 1 to hold. If $\beta>(<) 0$ and $\left(N_{j}-1\right) s^{C}+\frac{\beta+1}{\beta} s^{k}-\sum_{j} s^{i}>(<) 0$, this restriction is not needed for Lemma 1 to hold.

Turning to Proposition 1, after some algebra we get the equivalent of (16):

$$
s_{A, \min }-s_{B, \min }=\hat{s}_{A}^{m_{A}}-\hat{s}_{B}^{m_{B}}-\alpha\left(\frac{s^{C}}{s^{m_{A}}}\right)^{\beta}\left(\hat{s}_{A}-\hat{s}_{B}\right)+\left(\frac{1}{\left(s^{m_{A}}\right)^{\beta}}-\frac{1}{\left(s^{m_{B}}\right)^{\beta}}\right) \alpha W_{B} .
$$

In consequence, Proposition 1 holds but it depends on the adjusted condition:

$$
z \equiv \min \left\{1, \frac{\hat{s}_{A}^{m_{A}}-\hat{s}_{B}^{m_{B}}}{\left(\frac{s^{C}}{s^{m} A}\right)^{\beta}\left(\hat{s}_{A}-\hat{s}_{B}\right)+\left(\frac{1}{\left(s^{m} B\right)^{\beta}}-\frac{1}{\left(s^{m} A\right)^{\beta}}\right) W_{B}}\right\} .
$$

\footnotetext{
${ }^{12}$ As the only quantitative difference, $z$ in Proposition 1 would also depend on the level of $\delta$.

${ }^{13}$ Note that the superscript $k$ in $s^{k}$ denotes member $k$, whereas $\beta$ denotes the power of $s^{k}$.
} 
Note that Proposition 1.(i) holds with (B.5) as upper bound for $\beta>0$. For $\beta<0$, we have $\frac{1}{\left(s^{m} B\right)^{\beta}}<\frac{1}{\left(s^{m} A\right)^{\beta}}$ and, thus, (B.5) becomes the lower bound for Proposition 1.(i), and vice versa for Proposition 1.(ii). This implies that, for $\beta>0$, if $s^{C}$ is not very much lower than $s^{m_{A}}$, then $z$ decreases with increasing $\beta$ but $z$ may still be positive because $W_{B}$ has an upper bound, $T$. Proposition 1 then holds qualitatively.

\section{B.3 Positive assortative matching}

Here we consider the case where matches between pairs of faculty members are "localized", that is that high (low) status members are more likely to interact with other high (low) status members. This implies that matching between faculty members is positively assortative. Since the focus of this paper is not on the details of the matching procedure but on the effects of a certain matching rule on competition among faculties, we use a reduced form approach to get positive assortative matching. ${ }^{14}$ Moreover, we want to capture the idea that, at the point of time when faculties decide the offer they will make to a candidate, there is some uncertainty about the identity of a member's next research partner/match.

Members of faculty $j$ are ordered in rank where the member with the lowest status, $s^{\underline{n}}{ }^{j}$, has rank 0 and the member with the highest status, $s^{\bar{n}_{j}}$, has rank $N_{j}-1$. Member $k$ has rank $K$. For simplicity, we assume that status is proportional to rank within the faculty: $s^{k}=\underline{K}_{j}+\mu K$, where $\mu>0$ and $\underline{K}_{A}>\underline{K}_{B}$.

We capture positive assortative matching by assuming that the status utility member $k$ expects from membership in faculty $j, u_{j}^{k}$, follows a binomial distribution, where $u_{j}^{k} \sim$ $B(n, p)$. In our model, $p=\frac{K}{N_{j}}$ (capturing the localized matching effect) and $n=\sum_{j} s^{i}-s^{k}$ (capturing the expected payoffs from the collaboration effect). Hence, abstracting from wage effects, we model the utility realized by member $k$ in faculty $j$ as being distributed according to

$$
u_{j}^{k} \sim B\left(\sum_{j} s^{i}-s^{k}, \frac{K}{N_{j}}\right) .
$$

By definition, the binomial distribution has mean $n p$, which implies that, for risk-neutral members, the expected status utility of member $k$ corresponds to

$$
\hat{s}_{j}^{k}=\left(\sum_{j} s^{i}-s^{k}\right) \frac{K}{N_{j}} .
$$

If candidate $C$ enters faculty $j$, this has three effects on the utility of member $k$. First, it dilutes $k$ 's expected utility from collaborating with his pre-entry fellow members. Second, it increases $k$ 's expected utility because $k$ might be matched with $C$ in the future. Third, it decreases $k$ 's expected utility because the wage of $C$ can no longer be used to finance amenities for other faculty members. Therefore, the utility differential of member $k$, the analog of (6), is:

$$
\Delta_{j}^{k}=\frac{K}{N_{j}+1}\left(\sum_{j} s^{i}-s^{k}+s^{C}\right)-\frac{K}{N_{j}}\left(\sum_{j} s^{i}-s^{k}\right)-\alpha \frac{W_{j}}{N_{j}} .
$$

\footnotetext{
${ }^{14}$ For a detailed treatment of positive assortative matching equilibria, see Becker (1974), Kremer (1993), Legros and Newman (2007), or Damiano et al. (2010).
} 
Using $s^{k}=\underline{K}_{j}+\mu K$ and, consequently, $\sum_{j} s^{i}=N_{j} \underline{K}_{j}+\mu\left(0+\cdots+N_{j}-1\right)=N_{j} \underline{K}_{j}+$ $\mu\left(N_{j}-1\right) \frac{N_{j}}{2}$, we can rewrite (B.8):

$$
\Delta_{j}^{k}=\frac{K s^{C}}{N_{j}+1}+\frac{K\left(\mu K-\mu\left(N_{j}-1\right) \frac{N_{j}}{2}-\left(N_{j}-1\right) \underline{K}_{j}\right)}{N_{j}\left(N_{j}+1\right)}-\alpha \frac{W_{j}}{N_{j}} .
$$

Setting $\Delta_{j}^{k}$ equal to zero and solving for $s^{C}$ yields the minimum status that member $k$ would require from candidate $C$ if $k$ was faculty $j$ 's decision maker:

$$
s_{j, \text { min }}^{k}=\frac{1}{N_{j}}\left(\mu\left(N_{j}-1\right) \frac{N_{j}}{2}-\mu K+\left(N_{j}-1\right) \underline{K}_{j}\right)+\alpha \frac{W_{j}\left(N_{j}+1\right)}{K N_{j}} .
$$

$s_{j, \min }^{k}$ strictly and monotonically decreases in $K$, i.e., in rank within faculty $j$. Hence, the median voter theorem applies under majority voting. Moreover, this result proves that Lemma 1 holds for the positive assortative matching model: the required status of the candidate is lower the higher the rank of the pivotal member of the existing faculty.

In this setting, the status of faculty $j$ 's median member is $s^{m_{j}}=\underline{K}_{j}+\frac{N_{j}-1}{2}$. Thus, if the median member decides, we have:

$$
s_{j, \text { min }}^{m}=\frac{N_{j}-1}{N_{j}}\left(\underline{K}_{j}+\mu \frac{N_{j}-1}{2}\right)+2 \alpha \frac{W_{j}\left(N_{j}+1\right)}{N_{j}\left(N_{j}-1\right)} .
$$

Related to (B.8), candidate $C$ expects from entry in faculty $j$ :

$$
\frac{K_{j}^{C}}{N_{j}+1} \sum_{j} s^{i}+W_{j}=\frac{K_{j}^{C}}{N_{j}+1}\left(N_{j} \underline{K}_{j}+\mu N_{j} \frac{N_{j}-1}{2}\right)+W_{j},
$$

where $K_{j}^{C}=K_{j}^{C}\left(s^{C}\right)$ denotes $C$ 's status rank within faculty $j$. It follows that the indifference condition, that is the minimum wage that makes $C$ join faculty $A$ and not $B$, is:

$$
\begin{aligned}
W_{A}= & W_{B}-\left(\frac{K_{A}^{C}}{N_{A}+1}\left(N_{A} \underline{K}_{A}+\mu N_{A} \frac{N_{A}-1}{2}\right)\right. \\
& \left.-\frac{K_{B}^{C}}{N_{B}+1}\left(N_{B} \underline{K}_{B}+\mu N_{B} \frac{N_{B}-1}{2}\right)\right) .
\end{aligned}
$$

After the substitution of (B.13) in (B.11), we can compare the minimal status positions required by both faculties. To facilitate interpretation, we assume that the size of the faculties is equal: $N_{A}=N_{B}=N .{ }^{15}$ Then we can write:

$$
\begin{aligned}
s_{A, \min }^{m}-s_{B, \min }^{m}= & \frac{N-1}{N}\left(\underline{K}_{A}-\underline{K}_{B}\right) \\
& +\frac{\alpha}{N-1}\left(K_{B}^{C}\left(\underline{K}_{B}+\mu \frac{N-1}{2}\right)-K_{A}^{C}\left(\underline{K}_{A}+\mu \frac{N-1}{2}\right)\right) .
\end{aligned}
$$

\footnotetext{
${ }^{15}$ Simple inspection of (B.13) reveals that differences in faculty size would have to be substantial to outweigh differences in $K_{A}^{C} / K_{B}^{C}$ or $\underline{K}_{A} / \underline{K}_{B}$.
} 
In our baseline model, the key result, Proposition 2, depends on the validity of Proposition 1.(i), which requires that $s_{A, \min }>s_{B, \min }$. It is straightforward to analyze (B.14) regarding this condition:

- For $\alpha \rightarrow 0$, given that $\underline{K}_{A}>\underline{K}_{B}$, by definition, $s_{A, \min }>s_{B, \min }$. This means that, if the budget remaining after hiring cannot be used for other faculty amenities, the high status faculty $A$ will demand a strictly higher status from new members than will low status faculty $B$.

- For $\alpha>0$, given that $\underline{K}_{A}>\underline{K}_{B}$ but $K_{A}^{C}<K_{B}^{C}$ (the candidate would have to accept a lower rank in the high status faculty than in the low status faculty), the sign of the second term in (B.14) is ambiguous.

- For $\underline{K}_{A} K_{A}^{C}>\underline{K}_{B} K_{B}^{C}$, membership in faculty $A$ is so attractive for $C$ that he accepts a relatively low wage there, just as in our baseline model. This makes it cheaper for faculty $A$ to hire the candidate: $s_{A, \min }-s_{B, \min }$ sinks. As long as $\alpha$ is not too high, the negative effect of the second term in (B.14) does not outweigh the positive effect of the first term, though. Proposition 1.(i) holds qualitatively (with a slightly adjusted threshold value $z$ ).

- For $\underline{K}_{A} K_{A}^{C}<\underline{K}_{B} K_{B}^{C}$, the higher rank that the candidate can obtain in faculty $B$ outweighs the lower status of faculty $B$ members, from $C$ 's perspective. Then, both terms in (B.14) are positive, which implies that there is not even an upper threshold $z$ on $\alpha$.

We conclude that the positive assortative matching model even strengthens Proposition 1.(i) of our baseline model. All subsequent results thus also are robust.

\section{References}

Barbera, S., Maschler, M., \& Shalev, J. (2001). Voting for voters: a model of electoral evolution. Games and Economic Behavior, 37, 40-78.

Becker, G. S. (1974). A theory of marriage: Part II. Journal of Political Economy, 82, S11-S26.

Buchanan, J. M. (1965). An economic theory of clubs. Economica, 32, 1-14.

Cai, H., \& Feng, H. (2007). A theory of organizational dynamics: internal politics and efficiency (SSRN Working Paper).

Cornes, R., \& Sandler, T. (1996). The theory of externalities, public goods, and club goods. Cambridge: Cambridge University Press.

Couch, J. F., Shughart, W. F. II, \& Williams, A. L. (1993). Private school enrollment and public school performance. Public Choice, 76, 301-312.

Damiano, E., Li, H., \& Suen, W. (2010). First in village or second in Rome? International Economic Review, $51,263-288$.

Ellickson, B., Grodal, B., Scotchmer, S., \& Zame, W. R. (1999). Clubs and the market. Econometrica, 67, 1185-1217.

Epple, D., \& Romano, R. (1998). Competition between private schools, vouchers, and peer-group effects. American Economic Review, 62, 33-62.

Epple, D., \& Romano, R. (2008). Educational vouchers and cream skimming. International Economic Review, 49, 1395-1435.

Hansmann, H. (1986). A theory of status organizations. Journal of Law, Economics, and Organization, 2 , $119-130$.

Helsley, R. W., \& Strange, W. C. (1991). Exclusion and the theory of clubs. Canadian Journal of Economics, 24, 888-899.

Hoxby, C. M. (1996). Are efficiency and equity in school finance substitutes or complements? Journal of Economic Perspectives, 10, 51-72.

Kremer, M. (1993). The O-ring theory of economic development. Quarterly Journal of Economics, 108, 551-575.

Legros, P., \& Newman, A. F. (2007). Beauty is a beast, frog is a prince: assortative matching with nontransferabilities. Econometrica, 75, 1073-1102. 
Masten, S. E. (1995). Old school ties: financial aid coordination and the governance of higher education. Journal of Economic Behavior and Organization, 28, 23-47.

Masten, S. E. (2006). Authority and commitment: why universities, like legislatures, are not organized as firms. Journal of Economics and Management Strategy, 15, 649-684.

Olson, M. (1965). The logic of collective action. Cambridge: Harvard University Press.

Sandler, T., \& Tschirhart, J. (1980). The economic theory of clubs: an evaluative survey. Journal of Economic Literature, 18, 1481-1521.

Sobel, J. (2001). On the dynamics of standards. RAND Journal of Economics, 32, 606-623. 